CORRECTION

\title{
Correction to: The transcription factor KLF14 regulates macrophage glycolysis and immune function by inhibiting HK2 in sepsis
}

Yuan Yuan, Guangjian Fan (D), Yuqi Liu, Lu Liu, Tong Zhang, Pengfei Liu, Qing Tu, Xinyi Zhang, Shiyuan Luo, Liangfang Yao,

Feng Chen and Jinbao Li

(c) The Author(s), under exclusive licence to CSI and USTC 2022

Cellular \& Molecular Immunology (2022) 19:650; https://doi.org/10.1038/s41423-022-00839-4

Correction to: Cellular \& Molecular Immunology https://doi.org/ 10.1038/s41423-021-00806-5, published online 4 January 2022

In the version of this article initially published, two unintended errors were made during manuscript editing. The corresponding author's name was misspelled and therefore incorrectly listed as Jingbao Li with the email address lijingbaoshanghai@163.com. This author's name has been corrected to Jinbao Li with the email address lijinbaoshanghai@163.com. The results and conclusions were not affected. 